\title{
FACTORES QUE INTERVIENEN EN EL APRENDIZAJE DE LA QUÍMICA DESDE LAS REPRESENTACIONES SOCIALES DE LA JUVENTUD
}

\section{FACTORS INVOLVED IN LEARNING CHEMISTRY FROM THE SOCIAL REPRESENTATIONS OF YOUTH}

\author{
Juan Maria Torres ${ }^{1}$ \\ Luz Estela Romero Ramírez² \\ Mawency Vergel Ortega ${ }^{3}$ \\ Universidad Francisco de Paula Santander
}

\section{RESUMEN}

La investigación de carácter comprensivo tiene como propósito identificar factores que intervienen en el aprendizaje de la química desde las representaciones sociales de la juventud. Desde una perspectiva epistemológica, la investigación se fundamenta en la teoría de las representaciones sociales, utiliza los

1 Departamento de Química, Universidad Francisco de Paula Santander, Colombia. Ingeniero Químico, Magister en ciencia y técnica del Carbón. Orcid: https://orcid.org/0000-0003-3276-6273 Correo electrónico: juanmariatc@ufps.edu.co

2 Departamento de Química, Universidad Francisco de Paula Santander, Colombia. Orcid: https://orcid. org/0000-0002-5530-3776 Correo electrónico: luzestelarr@ ufps.edu.co

3 Departamento de Matemáticas y estadística Electrónica, Universidad Francisco de Paula Santander- Colombia. Orcid: https://orcid.org/0000-0001-8285-2968 Correo electrónico: mawency@ufps.edu.co procedimientos de análisis sistemático indicados en la metodología de la teoría fundamentada. Los resultados señalan que en las representaciones de los estudiantes intervienen factores como: preconcepciones, antecedentes académicos, papel del maestro como formador y ser humano, componente afectivo y actitudinal, el uso de representaciones para facilitar el aprendizaje y la reflexión crítica que realiza el estudiante sobre su proceso formativo. Se concluye que se deben gestionar labores conjuntas e interdisciplinarias para originar cambios desde que inicia la formación, de esta manera, la problemática del proceso enseñanza-aprendizaje de la química será superado 
Palabras clave: representaciones, química, profesor, aprendizaje

\section{ABSTRACT}

The purpose of this comprehensive research is to identify factors that intervene in the learning of chemistry from the social representations of youth. From an epistemological perspective, the research is based on the theory of social representations, using the systematic analysis procedures indicated in the grounded theory methodology. The results indicate that the students' representations are influenced by factors such as: preconceptions, academic background, the teacher's role as a trainer and human being, affective and attitudinal components, the use of representations to facilitate learning and the critical reflection made by the student on his or her formative process. It is concluded that joint and interdisciplinary work must be managed to bring about changes from the beginning of the training, thus, the problems of the teaching-learning process in chemistry will be overcome.

\section{KEY WORDS:}

representations, chemistry, teacher, learning

\section{INTRODUCCIÓN}

La investigación identifica y analiza las representaciones sociales acerca del aprendizaje de la química de los estudiantes repitentes, considerando los problemas de aprendizaje, el bajo rendimiento académico, la precognición de conceptos, la actitud negativa de los estudiantes hacia el aprendizaje del y la química, los altos índices de repitencia, las dificultades en la lectura, la escritura, la expresión oral, el análisis interpretativo, el papel del maestro como formador y la influencia de la tecnología (Zambrano, 2000). Con el fin de realizar el análisis interpretativo de las causas mencionadas para determinar los posibles resultados del estudio, se utiliza principalmente la teoría de las representaciones sociales enmarcada en la psicología social, la cual ha permitido indagar sobre diferentes líneas de investigación. Al mismo tiempo, se han establecido inquietudes acerca de cómo la realidad es construida por los sujetos y cómo influye la sociedad en la construcción, transformación y diversificación del conocimiento científico en los individuos. (Jodelet, 2003).

Las prácticas pedagógicas y los factores motivacionales del docente inciden en la repitencia de la química, por eso, tratarlas se convierte en uno de los objetivos principales de este trabajo. Así mismo, no puede desconocerse el componente ético de la comunidad universitaria, en particular del docente, quien tiene una importante función social y cultural en la construcción del joven estudiante y, por ende, de la sociedad. Otra característica importante del estudio consiste en analizar la realidad académica de los estudiantes que ingresan a primer semestre para formular planteamientos metodológicos interesantes, renovadores del sentido común, y los saberes previos que traen los jóvenes que muchas veces están llenos de errores. Lo anterior, con el fin de construir esa realidad y hacer que los conceptos y las aplicaciones del y la química sean más precisos y entendibles.

La propuesta ética de la pedagogía se manifiesta en la formación del hombre, su inserción en la cultura y su transformación en un individuo libre y autónomo. El reconocimiento de la existencia del otro como un ser en permanente formación, da sentido y significado al propósito esencial de la educación: construir un proyecto de humanidad (Campo y Restrepo, 2000).

\section{DISEÑO METODOLÓGICO}

Para construir esta reflexión de carácter comprensivo y pedagógico, se usa como línea de apoyo la teoría fundamentada para dinamizar los datos recolectados. Según Strauss y 
Corbin (2002), se "relacionan datos de manera sistemática y se analizan por medio de un proceso de investigación". Así mismo, Sandoval (1997), dice que: "es una metodología general para desarrollar una teoría a partir de datos que son sistemáticamente capturados y analizados; es una forma de pensar acerca de los datos y poderlos conceptuar". Los autores afirman que para construir conocimiento se debe seguir una metodología donde los datos obtenidos deben ser bien analizados y retroalimentarse, es decir, que sea cíclica y no lineal, pues sabemos dónde comenzamos la investigación, pero no dónde la terminamos.

La población está conformada por, aproximadamente, 150 estudiantes de la asignatura de y química en la carrera de Ingeniería Industrial; Los educandos están conformados por tres grupos: cada uno tiene alrededor de 45 a 50 estudiantes, estos datos son homologados por una participante de este proyecto que es docente de la asignatura, así como por otros docentes que dirigen los otros dos grupos. Se seleccionó una muestra de 46 estudiantes mediante un muestreo intencional a los que se les realizó una entrevista a profundidad e independiente a 25 de ellos, los otros 21 se organizaron en dos grupos focales de 7 y 14 , hasta la saturación teórica, que es el punto donde la información recopilada no produce aportes novedosos para la investigación. La entrevista se completó a medida que se desarrolló el trabajo de campo, se recolectó y se analizó la información.

El documento usado para la recolección de información es la entrevista semiestructurada. Strauss y Corbin (2002), y el grupo focal. Las fases que conforman el diseño de la investigación son: exploración, trabajo de campo, análisis de la información y producción del informe final. Estas etapas deben retroalimentarse una con otra para que el proyecto sea de calidad, es decir, el trabajo no debe ser lineal sino cíclico, pues al iniciarlo genera incertidumbre porque sus características todavía no se han formalizado. Cada fase tendrá documentos específicos que relacionen las actividades para controlar y supervisar la ejecución de las mismas. Además, estas permiten una rápida identificación de los distintos problemas y la adaptación a nuevas restricciones que surjan debido a circunstancias no previstas inicialmente.

\section{RESULTADOS}

El aprendizaje de la química en los jóvenes, se hace evidente a través de los resultados obtenidos en las estadísticas de los últimos cinco años, en promedio. Estas reportan que un $40 \%$ de los jóvenes deben repetir Química (2019), además de la tasa de retiro en programas de química $5 \%$. Si bien este porcentaje ha disminuido, las cifras aún son preocupantes para estudiante, los docentes, la familia, el plan de estudios y la misma universidad, comprometida en generar egresados altamente calificados, con competencias técnicas, pensamiento crítico e innovador y sentido de responsabilidad.

Del estudio toma interés en investigar por qué en la universidad existe alta repitencia, hecho que ocasiona la prolongación del tiempo de permanencia, dado que las materias mencionadas son asignaturas o ejes temáticos del ciclo básico. Así mismo, algunos jóvenes no logran alcanzar el promedio ponderado exigido por la institución, por lo que los educandos no pueden culminar sus estudios y abandonan la educación superior.

La investigación conduce a una visión conjunta de estudiantes, docentes y comunidad universitaria, con el fin de construir proyectos que contribuyan a la estructuración de ese ser humano fundamentado en el propósito de formación Los resultados sintetizan la reunión de los códigos substantivos; por último, la combinación y la reducción de estos permitieron establecer los siguientes códigos conceptuales: 
El papel del maestro en la formación integral, el estudiante en el proceso de construcción del conocimiento, el papel del componente afectivo y actitudinal en el proceso de aprendizaje, las representaciones sociales como ayuda en el aprendizaje, la visión del estudiante de su proceso formativo.

Contextualizando el proceso en el "aprendizaje significativo" $y$, finalmente, descubriendo la categoría central, aquella con mayor frecuencia que se conecta de buena manera con las categorías, la cual resultó "Formación de un ser humano". Los códigos conceptuales mencionados anteriormente son resultado de del análisis de las opiniones de los estudiantes, muestra como macrocategorías pedagogía crítica, proceso cognitivo, antecedentes, proceso de estudio, actitud, currículo, entorno ambiental y reflexión crítica

Tabla 1. Macrocategorías

\begin{tabular}{|c|c|}
\hline MACROCATEGORÍA & CATEGORÍAS SUBCATEGORÍAS \\
\hline $\begin{array}{l}\text { 1. PEDAGOGÍA Y } \\
\text { DIDÁCTICA }\end{array}$ & $\begin{array}{l}\text { PROCESOS Discurso pedagógico, estrategias de enseñanza, Actitud } \\
\text { del docente, Conciencia del docente, Transposición didáctica, Interacción } \\
\text { docente-estudiante, dinámica en la enseñanza, motivación, reflexión, } \\
\text { Herramientas, transmisión del discurso, planificación, evaluación,, } \\
\text { acompañamiento, Materiales didácticos, bagaje cultural, Saber disciplinar, } \\
\text { estructura teórica, vocación, apoyo, fortalecimiento de ideas }\end{array}$ \\
\hline $\begin{array}{l}\text { 2. PROCESO } \\
\text { COGNITIVO }\end{array}$ & $\begin{array}{l}\text { POCESOS DE COMPRENSIÓN Dificultades, formación, preparación, } \\
\text { poco entendimiento, falta de atención, Concentración, falencia en proceso } \\
\text { metacognitivo, poca comprensión } \\
\text { PROCESOS DE CONSTRUCCIÓN DEL CONOCIMIENTO Razonamiento, } \\
\text { Estructura teórica, Reelaboración conceptual, Retroalimentación de sus } \\
\text { representaciones, Procedimientos, Interpretación,Fortalecimiento de ideas, } \\
\text { Conocimiento previo }\end{array}$ \\
\hline 3. ANTECEDENTES & $\begin{array}{l}\text { FORMACIÓN Falta de conocimiento,Buenas bases,Pocos cimientos,Buena } \\
\text { educación, Malas bases conceptuales, poca fortaleza, Deficiencia, Poca } \\
\text { conceptualización }\end{array}$ \\
\hline $\begin{array}{l}\text { 4. PROCESO DE } \\
\text { ESTUDIO }\end{array}$ & $\begin{array}{l}\text { METODOLOGÍA Disciplina, Poca dedicación, conceptualización, } \\
\text { Integración, Percepción, aplicación de conceptos, Resolución de nuevos } \\
\text { problemas, Asesorías, Planeación, Talleres, Investigación, Apoyo y refuerzo } \\
\text { tecnológico, Formulación de preguntas, Organización }\end{array}$ \\
\hline 5. ACTITUD & $\begin{array}{l}\text { MOTIVACIÓN DEL ESTUDIANTE } \\
\text { Positiva, Entusiasmo, Gusto, Apatía, Interés, Compromiso, Consciencia, } \\
\text { Atención, Flexible, Ocasional, Indecisión } \\
\text { ACOMPAÑAMIENTO AFECTIVO DEL DOCENTE } \\
\text { Importancia, Motivación }\end{array}$ \\
\hline
\end{tabular}




\begin{tabular}{|c|c|}
\hline 6. CURRÍCULO & $\begin{array}{l}\text { APRENDIZAJE Significativo, crítico, Coherencia, Recursos, Aplicación, } \\
\text { Práctico, Dinámico, Tecnología } \\
\text { CRITERIOS Educación significativa, Educación práctica, Desarrollo de } \\
\text { competencias } \\
\text { COMPETENCIAS Habilidad, Destreza, Aplicación }\end{array}$ \\
\hline $\begin{array}{l}\text { 8. USO DE } \\
\text { REPRESENTACION }\end{array}$ & $\begin{array}{l}\text { ACERCAMIENTO DE LO ABSTRACTO A LA REALIDAD } \\
\text { Visión, Abstracción, Dificultad, Preconcepción, Percepción, Motivación, } \\
\text { Didáctica, Aprendizaje, Entendimiento, Experiencias, Aplicación, Relación, } \\
\text { Dificultad, Actividad en el aprendizaje, Práctica, Metodología, Estrategias } \\
\text { INFLUENCIA DE LA TECNOLOGÍA Distracción, juegos, doble } \\
\text { funcionalidad y multitareas, Globalización, Utilidad, Facilidad, Abstracción, } \\
\text { Ahorro de tiempo, Naturaleza de la opinión, Construcción de opinión, } \\
\text { Innovación, Actualización }\end{array}$ \\
\hline $\begin{array}{l}\text { 9. ENTORNO } \\
\text { AMBIENTAL }\end{array}$ & $\begin{array}{l}\text { VALORES Y RELACIONES INTERPERSONALESImportancia, } \\
\text { Atribuciones, Paciencia } \\
\text { AMBIENTE Concentración, Rendimiento, Bloqueo, Motivación, Atención, } \\
\text { Concentración, Interés, Apatía, Condiciones adecuadas, Silencio, } \\
\text { Intranquilidad }\end{array}$ \\
\hline $\begin{array}{l}\text { 10. REFLEXIÓN } \\
\text { CRÍTICA }\end{array}$ & $\begin{array}{l}\text { ORIENTACIÓNConductas, Reflexión, Influencia, Retroalimentación, } \\
\text { Fortalecimiento } \\
\text { ENSEÑANZA INTEGRALComprensión, Cumplimiento, Humanidad, } \\
\text { Respeto, Formación, Mejoramiento, Alternativa }\end{array}$ \\
\hline
\end{tabular}

El análisis de códigos sustantivos permiten observar que son categorías emergentes consideradas factores que influyen en el aprendizaje desde las representaciones sociales el proceso pedagógico, el proceso cognitivo, técnicas de estudio, percepción de la juventud, y la crítica constructiva. 


\section{ANÁLISIS DE CÓDIGOS SUBSTANTIVOS}

\begin{tabular}{|c|c|}
\hline $\begin{array}{l}\text { CÓDIGOS } \\
\text { SUBSTANTIVOS }\end{array}$ & INTERPRETACIÓN \\
\hline $\begin{array}{l}\text { PROCESO PEDAGÓGICO } \\
\text { Didáctica y currículo }\end{array}$ & $\begin{array}{l}\text { Metodología, Discurso pedagógico, Estrategias del docente } \\
\text { Actitud docente-estudiante, Dinámica en la enseñanza, bagaje cultural, } \\
\text { Fortalecimiento de ideas, Construcción del conocimiento, Aprendizaje } \\
\text { significativo -Influencia de la tecnología - Educación práctica - } \\
\text { competencias- habilidades }\end{array}$ \\
\hline $\begin{array}{l}\text { PROCESO COGNITIVO } \\
\text { Procesos cognitivos } \\
\text { Antecedentes }\end{array}$ & $\begin{array}{l}\text { Antecedentes académicos, positivos y negativos Falencia en proceso } \\
\text { metacognitivo- Análisis anticipados }\end{array}$ \\
\hline $\begin{array}{l}\text { ESTUDIO } \\
\text { Metodología de estudio } \\
\text { Actitud-Entorno ambiental }\end{array}$ & $\begin{array}{l}\text { Disciplina para estudiar -Percepción de sus compañeros -Aplicación } \\
\text { de conceptos -Dificultades en los estudios -Apoyo tecnológico- } \\
\text { Asesorías } \\
\text { Actitud ocasional para estudiar -Compromiso con el estudio-Lo } \\
\text { afectivo refuerza el conocimiento-El ambiente rinde o bloquea }\end{array}$ \\
\hline $\begin{array}{l}\text { PERCEPCIÓN } \\
\text { Uso de representaciones } \\
\text { Conocimiento cotidiano }\end{array}$ & $\begin{array}{l}\text { De la aplicación y la relación teórico-práctica } \\
\text { De las diferentes actividades realizadas por el docente, por sus } \\
\text { compañeros y por la misma institución-De la innovación en la } \\
\text { tecnología }\end{array}$ \\
\hline CRÍTICA CONSTRUCTIVA & $\begin{array}{l}\text { Orientación de conductas -Enseñanza integral -Mejoramiento en la } \\
\text { enseñanza -Comprensión de comportamiento-Los docentes deben } \\
\text { ser más humanos Retroalimentación estudiante-docente-estudiante }\end{array}$ \\
\hline
\end{tabular}

\section{CONCLUSIONES Y RECOMENDACIONES}

Los factores que influyen en el aprendizaje de la química en la juventud, desde sus representaciones sociales son el proceso pedagógico, el proceso cognitivo, técnicas de estudio, percepción de la juventud, y la crítica constructiva.

Elentendimientodelas representacionessociales de jóvenes universitarios y su influencia en la comprensión y el aprendizaje significativo del y la química, se basó en los resultados encontrados en las manifestaciones de los estudiantes con respecto a la formación académica básica con la que llegan los jóvenes a la universidad, el modo significativo de la enseñanza-aprendizaje en sus estudios superiores, las acciones pedagógicas desarrolladas por el programa y la universidad, la actitud y la motivación tanto del maestro como del estudiante en el aprendizaje significativo, los medios tecnológicos que intervienen en la formación integral de ese ser humano tan importante para la familia y la sociedad.

Al momento que los estudiantes ingresan a la educación superior se encuentran con una compleja realidad social, académica y cultural, su accionar los lleva a generar estrategias para su adaptación y permanencia, con el fin 
de culminar con éxito sus estudios. La mayoría de los jóvenes manifiestan que el grado de dificultad en el aprendizaje del y la química es por las estrategias pedagógicas y didácticas usadas por los maestros.

Las labores pedagógicas que deben desarrollar los profesores en beneficio de los jóvenes son el papel fundamental del maestro en la formación integral del estudiante, él posee un contacto directo con los jóvenes, además de la influencia en la mayoría de sus acciones pedagógicas. Por lo tanto, en el aprendizaje significativo desarrollado desde una mirada psicosocial, es fundamental tener en cuenta las representaciones sociales de docentes y estudiantes, que se ponen en juego en el aula de clase y que inciden en la práctica educativa (Aguilar y Mazzitelli, 2011).

\section{REFERENCIAS BIBLIOGRÁFICAS}

Abric, J.C. (1994a). Metodología de recolección de las representaciones sociales. Practiques sociales.

Aguilar, S. B., Mazzitelli, C. A., Chacoma, M. S., \& Aparicio, M. T. (2011). Saberes del docente y representaciones sociales: implicancias para la enseñanza de las ciencias naturales. Revista Electrónica" Actualidades Investigativas en Educación", 11(2), 1-28.

Arroyo, L. (2004). La implantación del enfoque constructivista en el aula de ciencia: estudio de caso múltiple. Universidad de Puerto Rico.

Ausubel, P. (1973). Algunos aspectos psicológicos de la estructura del conocimiento. En Elam, S. (comp.) La educación y la estructura del conocimiento. Investigaciones sobre el proceso de aprendizaje y la naturaleza de las disciplinas que integran el currículum. El Ateneo.

Chevallard, Y. (1991). La transposition didactique. Du savoir savant au savoir enseigné. (2a Edición en colaboración con Marie-Alberte Joshua). La Pensée Sauvage.

Clémence, A. (2001). Social positioning and social representations. In K. Deaux, \& G. Philogène (Eds.), Representations of the social (pp. 83-95). Oxford, UK, Malden, MA: Blackwell.

Durkheim, E. (1987). La división del trabajo social (Vol. 39). Ediciones Akal.

Echavarría, C. (2011). La escuela un escenario de socialización para la construcción de identidad moral. II Encuentro Internacional y $V$ nacional: Escuela, Familia y Medios, Escenarios para la Paz y el Desarrollo Humano. Encuentro llevado a cabo en Manizales, Colombia.

Elder, L. y Paul, R. (2004). Fundación para el Pensamiento Crítico. Revista El Educador.

lafrancesco, G. (2013). La investigación pedagógica formativa. Magisterio.

Jodelet, D. (2000). Representaciones sociales: contribución a un saber sociocultural sin fronteras. En D. Jodelet y A. Guerrero (eds.), Develando la cultura. Estudios en representaciones sociales, (7-30). UNAM.

Levina, E. (1987). De otro modo que ser, o más allá de la esencia. Sígueme. 
Mallart, J. (2000). Didáctica: del currículum a las estrategias de aprendizaje. Revista Española de Pedagogía, (217), 417438.

Martínez, A. (2006). Los conceptos de conocimiento, epistemología $y$ paradigma, como base diferencial en la orientación metodológica del trabajo de grado. Universidad de Oriente.

Ministerio de Educación Nacional (MEN). (2016). Deserción estudiantil en la educación superior colombiana. Elementos para su diagnóstico y tratamiento. Autor.

Largo, F., Rojas J.P. y Vergel-Ortega M. 2020. La empatía en el aprendizaje de los estudiantes de educación básica desde la perspectiva de la interacción social. Bogotá: Ecoe Ediciones.

Mora, M. (2002). Lateoría de las representaciones sociales de Serge Moscovici. Athenea Digital.(2), 1-25. http://www.raco.cat/index. php/Athenea/article/viewFile/34106/33945

Moscovici, S. (1991). La sicología social I. Paidós.

Mugny, G., \& Papastamou, S. (1986). Los estilos de comportamiento y su representación social. S. Moscovici, Psicología Social II. Pensamiento y vida social. Psicología Social y problemas sociales, 507.

Naranjo, I. (2012). Representaciones sociales de los estudiantes de la media vocacional sobre la matemática y la química. Universidad de Córdoba.

Nieto, F., Rojas, J.P. y Vergel-Ortega, M (2019). Impacto de estrategia peda'gogica basada en el aprendizaje creativo para estudiantes de ingeniería. Bogotá: Ecoe ediciones.
Osnaya, F. (2004). Las representaciones sociales de las unidades de servicios de apoyo a la educación superior. Universidad Autónoma de Barcelona.

Ovalles, G. y Urbina, J. (2013). Abandono y permanencia estudiantil en el programa de Ingeniería Industrial de la Universidad Francisco de Paula Santander: Análisis de factores pedagógicos. Universidad Francisco de Paula Santander.

Pascarella, E. y Terenzini, P. (2005). How College Affects Students: Findings and Insights from Twenty Years of Research. Volume 2. A third decade of research. Jossey- Bass.

Pineda, D. y Mota, Á. (2006). Cómo se articulan las concepciones epistemológicas y de aprendizaje con la práctica docente en el aula. Revista Mexicana de Investigación Educativa, 11(31), 1307-1335.

Pérez, A. (2011). La hermenéutica y los métodos de investigación en ciencias sociales. Estudio filosofía, (44), 9-37.

Rateau, P. y Mónaco, G. L. (2013). La teoría de las representaciones sociales: orientaciones conceptuales, campos de aplicaciones y métodos. Revista CES Psicología, 6(1), 22-42.

Romero, L., Torres, J. y Vergel, M. 2021. Representaciones sociales de estudiantes universitarios sobre el aprendizaje del cálculo y la química. Bogotá: Ecoe ediciones.

Strauss, A. y Corbin, J. (2002). Bases de la investigación cualitativa. Técnicas $y$ procedimientos para desarrollar la teoría fundamentada. Universidad de Antioquia. 
Tamayo, O. (2006). Representaciones semióticas y evolución conceptual en la enseñanza de las ciencias y las matemáticas. Revista Educación y Pedagogía, 18(45), 37-49.

Tinto, V. (2007). Research and Practice of Student Retention: What is next? Journal of College Student Retention, $8,1-19$

Urrutial, E. (2019). Rendimiento Académico $y$ contexto familiar en estudiantes universitarios. Revista $N^{\circ} 3$ Saberes Educativos, 166-177. Universidad Concepción de Chile.

Vera, H. (2002). Representaciones y clasificaciones colectivas. La teoría sociológica del conocimiento de Durkheim. Sociológica, 17(50), 103121.

Vergel, M. Rojas, J.P. y Urbina, J. 2020. Imaginarios de educación superior en la juventud sorda. Bogotá: Ecoe ediciones

Zambrano, A. (2000). Pedagogía, educabilidad y formación docente. Nueva Biblioteca Pedagógica. 